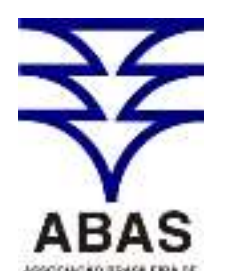

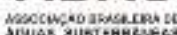
mww.abas.org

\title{
PANORAMA DO GERENCIAMENTO DE ÁREAS CONTAMINADAS NO BRASIL APÓS A RESOLUÇÃO CONAMA 420/09
}

\author{
OUTLOOK OF CONTAMINATED SITE MANAGEMENT IN BRAZIL AFTER \\ CONAMA 420/09 RESOLUTION
}

\author{
Antônia Angélica Correia de Araújo-Moura ${ }^{1}$ : Roberto Augusto Caffaro Filho ${ }^{2}$
}

Artigo recebido em: 10/07/2014 e aceito para publicação em: 26/07/2015.

DOI: http://dx.doi.org/10.14295/ras.v29i2.27972

\begin{abstract}
In industrialized countries, the discovery of the extent of the problem of soil and groundwater contamination led to the systematization of contaminated site management. After about five years of publication of CONAMA 420/09 Resolution, which established guidelines for contaminated site management in Brazil, the outlook of the country in relation to the theme was assessed in this study. Information was obtained from environmental agencies of each State through visits in their websites, telephone contacts and a questionnaire. The States of the southeast and south regions have made significant progress in management of soil contamination. On the other hand, in other regions of Brazil, contaminated site management is still in its infancy, and CONAMA 420/09 Resolution remains to be heeded. A qualitative assessment of the risk posed by the contamination of soil and groundwater in each State was performed, taking into account the level of management and the existing polluting potential. High level of risk was found in Paraná, Santa Catarina, Rio Grande do Sul, Espírito Santo, Distrito Federal, Ceará, Pernambuco and Bahia. The states of the northeast region undergoing rapid industrialization are in the most critical situation. It's necessary to advance quickly in contaminated site management, lest unjustifiable damage to the environment and public health that have occurred in industrialized regions of the country in past decades will be repeated. The advance of management in Brazilian States can be achieved through the creation of specific State laws and evolution of the technical level of professionals working in this area.
\end{abstract}

Keywords: Soil and groundwater contamination. Contaminated Site Management. CONAMA 420/09. Preliminary Hazard Analysis.

Resumo: Nos países industrializados, a descoberta da dimensão do problema da contaminação dos solos e das águas subterrâneas levou à sistematização do gerenciamento de áreas contaminadas. Cerca de cinco anos após a publicação da Resolução CONAMA 420/09, que estabeleceu diretrizes para o gerenciamento de áreas contaminadas no Brasil, foi levantado neste estudo o panorama do país em relacão ao tema. Foram obtidas informações junto aos órgãos ambientais de cada Estado através de consultas aos seus sítios eletrônicos, contatos telefônicos e aplicação de um questionário. Os Estados das regiões sudeste e sul avançaram significativamente no gerenciamento da contaminação do solo. Por outro lado, nas demais regiões do Brasil o gerenciamento de áreas contaminadas ainda é incipiente, e a Resolução CONAMA 420/09 ainda está por ser atendida. Foi realizada uma análise qualitativa do risco causado pela contaminação de solo e água subterrânea em cada unidade da federação, que levou em consideração o nível de gerenciamento praticado e o potencial poluidor existente. Foi encontrado nível de risco alto no Paraná, Santa Catarina, Rio Grande do Sul, Espírito Santo, Distrito Federal, Ceará, Pernambuco e Bahia. Os Estados da região nordeste em acelerado processo de industrialização encontram-se em situação mais crítica, sendo preciso avançar rapidamente no gerenciamento de áreas contaminadas, sob pena de repetirem-se os injustificáveis danos ao meio ambiente e à saúde pública ocorridos nas regiões industrializadas do país em décadas passadas. $\mathrm{O}$ avanço do gerenciamento nos Estados brasileiros pode ser alcançado com a criação de leis estaduais específicas e com a evolução do nível técnico dos profissionais que atuam nessa área.

Palavras-chave: Contaminação do Solo e Água Subterrânea. Gerenciamento de Áreas Contaminadas. CONAMA 420/2009. Análise Preliminar de Perigo.

1 Programa de Pós-Graduação em Recursos Hídricos e Saneamento, Universidade Federal de Alagoas (angelica.correia@hotmail.com)

${ }^{2}$ Centro de Tecnologia, Universidade Federal de Alagoas (roberto@ctec.ufal.br) 


\section{INTRODUÇÃO}

O solo é utilizado como destino final para os resíduos humanos desde os tempos pré-históricos. À medida que o homem foi evoluindo e se constituindo em sociedade, a contaminação passou de difusa a pontual. A Revolução Industrial iniciada no século XVIII representou mudanças na composição das substâncias depositadas no solo, e os contaminantes no meio ambiente aumentaram drasticamente devido ao desenvolvimento industrial e tecnológico ocorrido no século XX (SWARTJES, 2011).

A descoberta da dimensão do problema das áreas contaminadas e a pressão da sociedade levaram os países desenvolvidos, começando por Holanda e EUA, a desenvolverem diferentes respostas para a problemática da contaminação do solo (RODRIGUES JR., 2003). Convencionou-se chamar de 'gerenciamento de áreas contaminadas' o conjunto de medidas que asseguram o conhecimento das características dessas áreas e dos impactos causados pelas substâncias químicas ali presentes, sobretudo, para as águas subterrâneas, proporcionando os instrumentos necessários à tomada de decisão quanto às formas de intervenção mais adequadas.

A Holanda desenvolveu inicialmente sua política de gerenciamento de áreas contaminadas com o intuito de devolver ao solo suas características originais, preservando sua multifuncionalidade. A política holandesa evoluiu ao longo de três décadas, passando de um processo de gerenciamento rígido, para um processo maleável, seguindo o princípio "simples, se possível, complexo quando necessário", finalmente adotando o conceito de adequação do solo ao uso pretendido (SWARTJES et al., 2012).

Os EUA também possuem pioneirismo no gerenciamento de áreas contaminadas em relação aos demais países desenvolvidos, tendo sido impulsionados por casos emblemáticos de contaminação do solo como, por exemplo, o de Love Canal. A United States Environmental Protection Agency (U.S.EPA) é o órgão ambiental que conduz e supervisio- na ações de remediação de áreas contaminados nos EUA. Os EUA também utilizam atualmente o conceito de adequação do solo ao uso pretendido nos casos de remediação de solo contaminado.

Panagos et al., (2013) em revisão sobre a política ambiental da União Europeia perceberam que em 2001, a European Environment Agency (EEA) começou a desenvolver um conjunto de indicadores políticos ambientais, entre os quais um relacionado ao gerenciamento de áreas contaminadas. O objetivo deste indicador é quantificar o progresso na gestão de contaminações locais, identificar os setores que mais contribuem com contaminação do solo, classificar os principais contaminantes e abordar questões relacionadas com os investimentos na remediação dos solos. Com sua aplicação, a produção industrial foi apontada como principal responsável pela contaminação do solo (60\%) na Europa, seguida do setor de serviços $(33 \%)$ e mineração $(7 \%)$.

A União Europeia e os EUA buscam um gerenciamento de forma que a contaminação do solo não produza efeitos significativos à saúde humana e ao ecossistema. Para isso utilizam ferramentas padronizadas de avaliação de risco, considerando o uso pretendido do solo.

O gerenciamento de áreas contaminadas no Brasil começou a ser praticado inicialmente na região sudeste. A Companhia Ambiental do Estado de São Paulo (CETESB) por muitos anos desenvolveu uma metodologia baseada no modelo holandês, apoiada pela GTZ - Agência de Cooperação Alemã. Porém, no restante do país, a situação não era semelhante. Magalhães (2000) comparou aspectos do gerenciamento de áreas contaminadas praticado no Brasil em relação ao observado nos EUA, Canadá e países da Europa, e observou que no final da década de 1990 havia ainda uma atuação mínima no gerenciamento de áreas contaminadas brasileiro. Detectou falta de uma legislação específica, de valores de intervenção para o solo, de um registro oficial de áreas contaminadas e de uma estrutura provedora de fundos para 
a remediação de sítios abandonados.

A Resolução CONAMA n-420, de 28 de Dezembro de 2009, dispõe sobre critérios e valores orientadores de qualidade do solo (valores de prevenção e valores de intervenção) quanto à presença de substâncias químicas e estabelece diretrizes para o gerenciamento ambiental de solos contaminados por essas substâncias em decorrência de atividades antrópicas. Sua publicação significou um enorme avanço para as questões relacionadas ao gerenciamento de áreas contaminadas no Brasil, pois o Conselho Nacional do Meio Ambiente possuía resoluções para tratar da qualidade da água e do ar desde a década de 80, mas nada em relação à qualidade do solo (MATTIASO, 2010). Os critérios contidos nesta resolução são similares aos critérios praticados atualmente nos países desenvolvidos, incorporando a avaliação de risco e o uso pretendido do solo.

Em nível estadual, São Paulo, Minas Gerais e Rio de Janeiro possuem legislação referente ao gerenciamento de áreas contaminadas. São elas respectivamente: Lei $\mathrm{n}^{\circ}$ 13.577, de 08 de Julho 2009; Deliberação Normativa Conjunta COPAM/CERH n ${ }^{\circ}$ 02, de 08 de setembro de 2010 e Resolução CONEMA $\mathrm{n}^{\circ} 44$ de 14 de Dezembro de 2012.

De acordo com a Resolução CONAMA 420/09, os órgãos ambientais competentes deverão dar publicidade às informações sobre as áreas contaminadas identificadas e suas principais características. Ao Instituto Brasileiro de Meio Ambiente IBAMA cabe sistematizar tais informações dos Estados e disponibilizá-las em seu portal institucional na forma de um Banco de Dados Nacional sobre Áreas Contaminadas. Atualmente só estão disponíveis informações referentes aos três Estados que possuem legislação sobre o assunto (IBAMA, 2015).

O objetivo deste trabalho foi levantar o panorama do gerenciamento de áreas contaminadas praticado no Brasil, após cerca de cinco anos da publicação da Resolução CONAMA 420/09. Intencionou-se avaliar o nível do gerenciamento em todos os Estado- da Federação, especialmente em relação à aplicação das diretrizes que foram estabelecidas na Resolução. Também foi realizada uma análise qualitativa do risco causado pela contaminação de solo em cada unidade da federação, que levou em consideração o nível de gerenciamento praticado no Estado, o potencial poluidor existente e sua densidade demográfica.

\section{MATERIAIS E MÉTODOS}

\subsection{Coleta de Dados sobre o Gerenciamento de Áreas Contaminadas nos Estados}

Os dados sobre o gerenciamento de áreas contaminadas nos Estados foram coletados através de consultas aos sítios eletrônicos dos órgãos ambientais estaduais, consultas telefônicas e comunicações por correio eletrônico. O objetivo da consulta aos sítios eletrônicos dos órgãos ambientais foi o de verificar a existência e disponibilidade do cadastro das áreas contaminadas previsto na Resolução CONAMA 420/09. Ainda na página virtual de cada órgão ambiental buscouse verificar a existência de setor/servidor específico para tratar do assunto em questão. A necessidade de efetuar contato telefônico surgiu a partir da dificuldade em encontrar informações nos sítios eletrônicos. Também foi utilizado o contato por correio eletrônico para obtenção de resultados. Para padronizar as informações sobre o gerenciamento de áreas contaminadas praticado por cada órgão estadual foi elaborado um questionário com 7 perguntas (indicadas mais a frente na seção Resultados) baseadas em critérios e conceitos presentes na Resolução CONAMA 420/09. Além de respostas positivas ou negativas, os órgãos ambientais puderam assinalar 'em andamento' para qualquer uma das perguntas do questionário. O nível de gerenciamento de áreas contaminadas em cada Estado foi determinado através das respostas ao questionário. Como proposta metodológica deste trabalho, para cada resposta afirmativa foram atribuídos 2 pontos. Para cada resposta 'em andamento', foi atribuído 1 ponto. Nenhum ponto foi atribuído às respostas negativas. A 
pontuação obtida por cada Estado foi utilizada para enquadramento em uma das três categorias possíveis de nível de gerenciamento de áreas contaminadas (alto, médio e baixo). Pontuação maior que 9 foi considerada como nível alto de gerenciamento de áreas contaminadas. Pontuação menor que 4 foi considerada como nível baixo de gerenciamento e pontuação entre 4 e 9, nível médio de gerenciamento de áreas contaminadas.

\subsection{Análise de risco da ocorrência de im- pactos relacionados à contaminação do so- lo (RI)}

Com base na ferramenta denominada Análise Preliminar de Perigo - APP (CAMACHO, 2004), a cada Estado foi atribuído um nível de risco inerente à ocorrência de impactos relacionados à contaminação do solo. Tal risco está relacionado com o nível de industrialização e a densidade demográfica. Quanto maiores o nível de industrialização e a densidade demográfica, maior o risco. Nessa análise, não foi levada em consideração a contaminação decorrente de vazamentos em postos de combustíveis, mas apenas a participação dos Estados no parque industrial de transformação brasileiro. Informações sobre o desenvolvimento industrial dos Estados foram obtidas da Federação das Indústrias do Estado de São Paulo (FIESP, 2014) e suas respectivas densidades demográficas, do Instituto Brasileiro de Geografia e Estatística (IBGE, 2011). Para determinação de R1, o nível de industrializa- ção teve peso maior do que a densidade demográfica. Maior detalhamento na metodologia para determinação dos níveis de industrialização e densidade demográfica, bem como de R1, pode ser encontrado em Araújo (2014).

\subsection{Análise do risco relacionado ao nível de gerenciamento de áreas contaminadas nos Estados (R2)}

$\mathrm{Na}$ análise qualitativa de risco $\mathrm{R} 2$ foram cruzados o risco da ocorrência de impactos relacionados à contaminação do solo (R1), com o nível de gerenciamento de áreas contaminadas encontrado em cada Estado. Dessa forma, procurou-se aplicar uma ponderação da situação de cada ente federativo em relação à problemática das áreas contaminadas que levasse em conta tanto o potencial de danos à saúde pública e ao meio ambiente, quanto o nível do gerenciamento de áreas contaminadas praticado no Estado. A Tabela 1 mostra a matriz de cruzamento de dados para a análise de risco $\mathrm{R} 2$.

\section{RESULTADOS E DISCUSSÃO}

Na Figura 1 são mostradas, por Estado, as respostas ao questionário aplicado para avaliar o nível do gerenciamento de áreas contaminadas praticado pelos órgãos ambientais estaduais. De acordo com a resposta para cada pergunta, os Estados são apresentados em quatro cores diferentes.

Tabela 1 - Matriz de Classificação de Risco R2, cruzando o nível de gerenciamento de áreas contaminadas observado no Estado com o risco da ocorrência de impactos relacionados à contaminação do solo (R1)

Table 1 - Risk Rating Matrix R2, crossing the level of contaminated site management observed in the State with the risk of occurrence of impacts related to soil contamination (R1)

\begin{tabular}{|c|c|c|c|c|}
\hline \multirow{2}{*}{ Matriz de Classificação de Risco R2 } & \multicolumn{2}{|c|}{ Nível de gerenciamento de áreas contaminadas } \\
\cline { 2 - 5 } Nível de Risco R1 & Baixo & Alto & Médio & Baixo \\
\cline { 2 - 5 } & Médio & & & \\
\cline { 2 - 4 } & Alto & & & \\
\hline
\end{tabular}

Legenda - Verde: Risco Baixo. Amarelo: Risco Médio. Vermelho: Risco Alto 


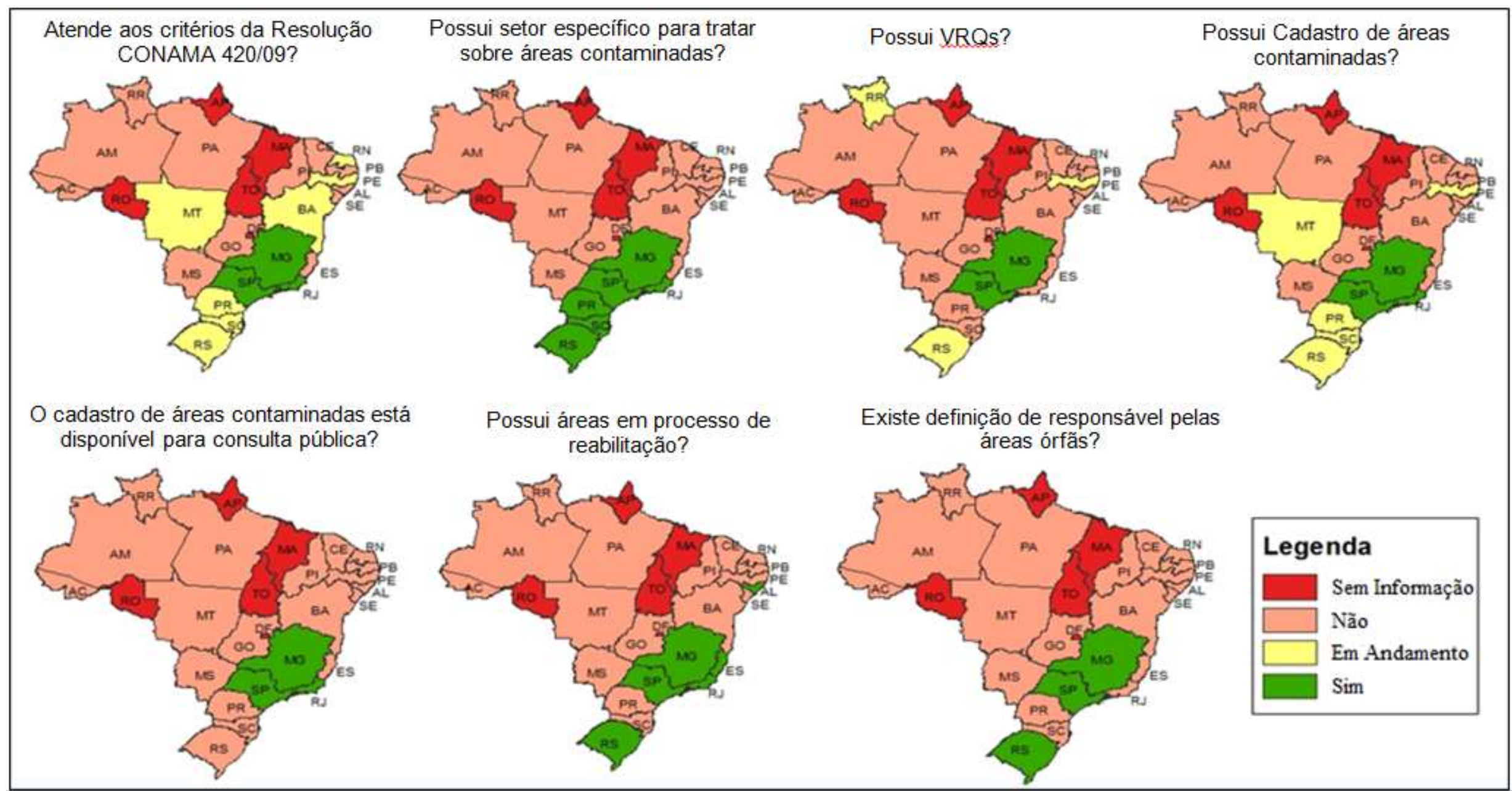

Figura 1 - Respostas ao questionário aplicado aos órgãos ambientais para avaliar o nível do gerenciamento de áreas contaminadas em cada Estado Figure 1 - Answers to the questionnaire applied to State environmental agencies to assess the level of contaminated site management in each State 
Vermelho escuro foi utilizado para identificar os Estados onde não foi possível obter nenhuma informação sobre a prática de gerenciamento de áreas contaminadas, seja através de consultas aos sítios eletrônicos dos respectivos órgãos ambientais, seja por contato telefônico com servidores do mesmo. Nos órgãos ambientais destes Estados, não foi possível localizar um setor ou servidor em condições de responder ao questionário. Tal situação ocorreu com os Estados do Amapá, Distrito Federal, Maranhão, Rondônia e Tocantins.

Nove Estados (Acre, Amazonas, Ceará, Goiás, Mato Grosso do Sul, Mato Grosso, Pará, Paraíba e Sergipe) responderam não a todas as perguntas do questionário (indicado pela cor vermelho claro), o que indica que nestes Estados, o gerenciamento de áreas contaminadas, caso exista, não segue os critérios da Resolução CONAMA 420/09.

Com base nesses resultados, pode-se afirmar que em metade dos Estados do Brasil ainda não foi implantada uma metodologia de gerenciamento de áreas contaminadas baseada nos critérios da Resolução CONAMA 420/09, mesmo após cerca de cinco anos de sua publicação. Uma explicação possível pode ser a carência, em muitos Estados, de profissionais com nível técnico suficiente para a consolidação das práticas exigidas pela Resolução, tanto na esfera pública quanto na iniciativa privada.

Por outro lado, os órgãos ambientais dos Estados de São Paulo e Minas Gerais responderam sim a todos os itens do questionário (indicado pela cor verde), demonstrando que o gerenciamento de áreas contaminadas nesses estados é baseado em critérios presentes na legislação federal. Outro Estado que se destacou por seu nível de gerenciamento é o Rio de Janeiro, que respondeu não a apenas dois itens do questionário. Vale ressaltar que esses três Estados já possuem leis estaduais relacionadas à matéria, o que por óbvio, fez avançar o tema.

Observa-se que os Estados de São Paulo, Minas Gerais e Rio de Janeiro são os únicos que possuem um cadastro de áreas contaminadas disponível para consulta pública. São Paulo iniciou o cadastramento em maio de 2002, registrando 255 áreas suspeitas de contaminação. Em dezembro de 2013, havia 4.771 registros no Cadastro de Áreas Contaminadas e Reabilitadas no Estado de São Paulo (CETESB, 2013). O Estado de Minas Gerais publicou sua primeira lista de áreas contaminadas em 2009, totalizando 273 áreas suspeitas de contaminação. Em 2012 já registrava 365 declarações de áreas suspeitas de contaminação (FEAM, 2012). O Rio de Janeiro publicou sua primeira lista no final de 2013 com 160 áreas suspeitas de contaminação (INEA, 2014).

As regiões sudeste e sul apresentaram em geral melhor nível de gerenciamento de áreas contaminadas. Nesta última, o melhor exemplo é o Estado do Rio Grande do Sul, que apesar de ainda não possuir lei estadual, dispõe de uma Diretriz Técnica ( $\mathrm{N}^{0}$ 01/2011DIRTEC/FEPAM) relacionada ao assunto.

Na Tabela 2 é apresentada a classificação dos Estados em relação ao nível de gerenciamento de áreas contaminadas praticado pelo órgão ambiental, determinado através da pontuação obtida pelas respostas ao questionário.

$\mathrm{Na}$ Tabela 3 é apresentado o resultado da análise de risco da ocorrência de impactos ambientais relacionados à contaminação do solo (R1), que levou em consideração a industrialização e densidade demográfica nos Estados do Brasil.

De acordo com a análise, o risco de ocorrência de impactos ambientais relacionados à contaminação do solo (R1) é maior nos Estados das regiões sul e sudeste, que são as mais industrializadas do país. A região nordeste é uma região em processo de industrialização que apresenta em geral densidades demográficas elevadas, o que já levou alguns estados a apresentarem R1 médio. Dada a continuidade desse processo de industrialização, R1 tende a elevar-se em um futuro próximo na maioria dos Estados do nordeste. 
Tabela 2 - Nível de gerenciamento de áreas contaminadas nos Estados do Brasil, de acordo com a pontuação obtida pelas respostas ao questionário

Table 2 - Level of contaminated site management in the States of Brazil, according to the score obtained by the responses to the questionnaire

\begin{tabular}{lc}
\hline \multicolumn{1}{c}{ Estado } & $\begin{array}{c}\text { Nível de gerencia- } \\
\text { mento de áreas con- } \\
\text { taminadas }\end{array}$ \\
\hline Região Sul & Médio \\
Paraná & Médio \\
Rio Grande do Sul & Médio \\
Santa Catarina & \\
Região Sudeste & Baixo \\
Espírito Santo & Alto \\
Minas Gerais & Alto \\
Rio de Janeiro & Alto \\
São Paulo & \\
Região Norte & Baixo \\
Acre & Baixo \\
Amazonas & Baixo \\
Amapá & Baixo \\
Pará & Baixo \\
Rondônia & Baixo \\
Roraima & Baixo \\
Tocantins & \\
Região Nordeste & Baixo \\
Alagoas & Baixo \\
Bahia & Baixo \\
Ceará & Baixo \\
Maranhão & Baixo \\
Paraíba & Baixo \\
Pernambuco & Baixo \\
Piauí & Baixo \\
Rio Grande do Norte & Baixo \\
Sergipe & Baixo \\
Região Centro - Oeste & Baixo \\
Distrito Federal & Baixo \\
Mato Grosso & Baixo \\
Mato Grosso do Sul & \\
Goiás & \\
\hline & \\
\hline
\end{tabular}

$\mathrm{Na}$ Tabela 4 é apresentada a classificação dos Estados em relação em ao risco $\mathrm{R} 2$, que é função do cruzamento de R1 com o nível do gerenciamento de áreas contaminadas encontrado em cada Estado.

Foi encontrado risco R2 alto nos Estados do Paraná, Rio Grande do Sul, Santa
Catarina, Espírito Santo, Bahia, Ceará, Pernambuco e Distrito Federal. Isso indica que nestes Estados a problemática da contaminação do solo demanda atenção especial. No entanto, há diferenças significativas entre os Estados pertencentes a esse grupo, que serão discutidas a seguir. 
Tabela 3 - Classificação dos Estados do Brasil em relação à análise de risco R1, de acordo com seu nível de industrialização e densidade demográfica

Table 3 - Classification of Brazilian States in relation to risk assessment R1, according to its level of industrialization and population density

\begin{tabular}{|c|c|c|c|}
\hline Estado & $\begin{array}{l}\text { Nível de Industria- } \\
\text { lização }\end{array}$ & $\begin{array}{c}\text { Densidade Demo- } \\
\text { gráfica }\end{array}$ & Risco R1 \\
\hline \multicolumn{4}{|l|}{ Região Sul } \\
\hline Paraná & Alto & Média & Alto \\
\hline Rio Grande do Sul & Alto & Média & Alto \\
\hline Santa Catarina & Alto & Média & Alto \\
\hline \multicolumn{4}{|l|}{ Região Sudeste } \\
\hline Espírito Santo & Médio & Média & Médio \\
\hline Minas Gerais & Alto & Baixa & Alto \\
\hline Rio de Janeiro & Médio & Alta & Alto \\
\hline São Paulo & Alto & Alta & Alto \\
\hline \multicolumn{4}{|l|}{ Região Norte } \\
\hline Acre & Baixo & Baixa & Baixo \\
\hline Amazonas & Baixo & Baixa & Baixo \\
\hline Amapá & Baixo & Baixa & Baixo \\
\hline Pará & Baixo & Baixa & Baixo \\
\hline Rondônia & Baixo & Baixa & Baixo \\
\hline Roraima & Baixo & Baixa & Baixo \\
\hline Tocantins & Baixo & Baixa & Baixo \\
\hline \multicolumn{4}{|l|}{ Região Nordeste } \\
\hline Alagoas & Baixo & Média & Baixo \\
\hline Bahia & Médio & Baixa & Médio \\
\hline Ceará & Médio & Média & Médio \\
\hline Maranhão & Baixo & Baixa & Baixo \\
\hline Paraíba & Baixo & Média & Baixo \\
\hline Pernambuco & Médio & Média & Médio \\
\hline Piauí & Baixo & Baixa & Baixo \\
\hline Rio Grande do Norte & Baixo & Média & Baixo \\
\hline Sergipe & Baixo & Média & Baixo \\
\hline \multicolumn{4}{|l|}{ Região Centro-Oeste } \\
\hline Distrito Federal & Baixo & Alta & Médio \\
\hline Mato Grosso & Baixo & Baixa & Baixo \\
\hline Mato Grosso do Sul & Baixo & Baixa & Baixo \\
\hline Goiás & Médio & Baixa & Médio \\
\hline
\end{tabular}


Tabela 4 - Classificação dos Estados do Brasil em relação à análise de risco R2, que levou em conta o nível de gerenciamento de áreas contaminadas praticado nos Estados e a análise de risco R1

Table 4 - Classification of Brazilian States in relation to risk analysis R2, according to the level of contaminated site management practiced and R1 risk assessment

\begin{tabular}{lr}
\hline \multicolumn{1}{c}{ Estado } & Risco R \\
\hline Região Sul & Alto \\
Paraná & Alto \\
Rio Grande do Sul & Alto \\
Santa Catarina & \\
Região Sudeste & Alto \\
Espírito Santo & Médio \\
Minas Gerais & Médio \\
Rio de Janeiro & Médio \\
São Paulo & \\
Região Norte & Médio \\
Acre & Médio \\
Amazonas & Médio \\
Amapá & Médio \\
Pará & Médio \\
Rondônia & Médio \\
Roraima & Médio \\
Tocantins &
\end{tabular}

\section{Região Nordeste}

\begin{tabular}{lc} 
Alagoas & Médio \\
Bahia & Alto \\
Ceará & Alto \\
Maranhão & Médio \\
Paraíba & Médio \\
Pernambuco & Alto \\
Piauí & Médio \\
Rio Grande do Norte & Médio \\
Sergipe & Médio \\
Região Centro-Oeste & \\
Distrito Federal & Alto \\
Mato Grosso & Médio \\
Mato Grosso do Sul & Médio \\
Goiás & Médio \\
\hline
\end{tabular}

Na região sul, todos os Estados apre- que explica o alto risco R2 encontrado. Posentaram nível médio de gerenciamento de rém, em nossa pesquisa observamos que há áreas contaminadas e alto nível de industria- uma série de ações em desenvolvimento por lização (e consequentemente, do risco R1), o parte dos órgãos ambientais nos Estados des- 
sa região. Provavelmente o nível de gerenciamento nesses Estados passará de médio para alto em um futuro próximo. Isso será suficiente para que neles o risco R2 seja diminuído para médio, deixando-os em situação similar aos Estados de São Paulo, Rio de Janeiro e Minas Gerais: altamente industrializados, mas com um nível alto de gerenciamento de áreas contaminadas.

O Espírito Santo destacou-se negativamente na região sudeste. Apresenta nível médio de industrialização, com um gerenciamento de áreas contaminadas de nível baixo. Já o Distrito Federal apresentou risco R2 alto devido a sua altíssima densidade populacional, somada a um nível baixo de gerenciamento de áreas contaminadas. É preciso melhorar substancialmente o gerenciamento nestes Estados.

Os Estados da Bahia, Ceará e Pernambuco apresentam situações bastante similares. São Estados em processo de industrialização acelerada (atualmente em nível R1 médio), mas onde ainda se pratica um nível baixo de gerenciamento de áreas contaminadas, sem indícios de melhora a curto prazo. Portanto, consideramos que estes Estados encontram-se em situação mais crítica que os demais.

O panorama do gerenciamento de áreas contaminadas no Brasil encontrado neste estudo deve ser comparado com aquele encontrado por Magalhães (2000) no final da década de 1990. É possível observar que os Estados das regiões sul e sudeste avançaram significativamente no gerenciamento da contaminação do solo. Ressaltamos que não fize-

\section{REFERÊNCIAS}

ARAÚJO, A. A. C. O Brasil no Contexto do Gerenciamento de Áreas Contaminadas: Um Olhar Crítico para as Regiões Desiguais do País. 2014. 32 p. Dissertação (Mestrado em Recursos Hídricos e Saneamento). UFAL, Maceió - AL.

BRASIL. Conselho Nacional do Meio Ambiente CONAMA. Resolução n ${ }^{\circ} 420$, de 28 de dezembro de 2009. Dispõe sobre critérios e valores orientadores de qualidade do solo quanto à presença de substâncias químicas e estabelece diretrizes para o gerenciamento ram mais do que sua obrigação, por se tratarem dos Estados mais ricos e desenvolvidos do país, onde atuam profissionais de nível técnico avançado nessa temática.

Por outro lado, nas demais regiões do Brasil o gerenciamento de áreas contaminadas ainda é incipiente. Nelas encontramos praticamente o mesmo quadro observado a 15 anos atrás (MAGALHÃEES, 2000). Porém, com o agravante de há cerca de cinco anos contarmos com uma legislação federal sobre o assunto, que ainda está por ser atendida.

\section{CONCLUSÃO}

Os resultados deste estudo demonstram que na região nordeste é preciso avançar muito rapidamente nas práticas de gestão da qualidade do solo e água subterrânea, sob pena de repetirem-se os injustificáveis danos ao meio ambiente e à saúde pública ocorridos nas regiões industrializadas do país em décadas passadas. $\mathrm{O}$ avanço do gerenciamento de áreas contaminadas nos Estados brasileiros pode ser alcançado com a criação de leis estaduais específicas e com a evolução do nível técnico dos profissionais que atuam na área, tanto na esfera pública quanto no setor privado.

\section{AGRADECIMENTOS}

Este trabalho foi apoiado pela Coordenação de Aperfeiçoamento de Pessoal de Nível Superior (CAPES) por meio de uma bolsa de mestrado concedida à Antônia Angélica Correia de Araújo.

ambiental de áreas contaminadas por essas substâncias em decorrência de atividades antrópicas. Diário Oficial da República Federativa do Brasil, Poder Executivo, Brasília, DF, 30 dez. 2009. Seção 1, 20p.

CAMACHO, E. N. Uma Proposta de Metodologia para Análise Quantitativa de Riscos Ambientais. 2004. 140 p. Dissertação (Mestrado em Ciências em Engenharia Civil). COPPE/UFRJ, Rio de Janeiro - RJ.

CETESB - Companhia Ambiental do Estado de São 
Paulo. Relação de Áreas Contaminadas e Reabilitadas do Estado de São Paulo. São Paulo: Diretoria de Controle e Licenciamento Ambiental da CETESB, 2013. 14p. Disponível em <http://www.cetesb.sp.gov.br/areascontaminadas/rela $\%$ E7\%F5es-de-\%E1\%A1reas contaminadas/4-rac> . Acesso em: dez. 2013.

FEAM - Fundação Estadual do Meio Ambiente. Inventário de áreas contaminadas do Estado de Minas Gerais - 2012. Governo do Estado de Minas Gerais, Belo Horizonte, 2012. 19 p. Disponível em: $<$ http://www.feam.br/noticias/1/1231--feam-publicainventario-de-areas-contaminadas $>$. Acesso em: nov. 2013.

FIESP - Federação das Indústrias do Estado de São Paulo. Panorama da Indústria de Transformação Brasileira. $3^{\text {a }}$ Ed. São Paulo: Departamento de Pesquisas e Estudos Econômicas - DEPECON, última atualização 07 de março de 2014.59 p.

IBAMA - Instituto Brasileiro de Meio Ambiente. Informações sobre o Banco de Dados Nacional sobre Áreas Contaminadas - BDNAC. Brasil. Disponível em: <http://www.ibama.gov.br/areastematicas-qa/areas-contaminadas $>$. Acesso em: mai. 2015.

IBGE - Instituto Brasileiro de Geografia e Estatística. Censo Demográfico 2010. Rio de Janeiro: IBGE, 2011. Disponível em: <http://http://www.ibge.gov.br/estadosat/perfil.php>. Acesso em: jan. 2014.

INEA - Instituto Estadual do Ambiente. Gerenciamento de Áreas Contaminadas do Estado do Rio Janeiro: Cadastro de Áreas Contaminadas e Reabilitadas. $1^{\text {a }}$ Ed. Governo do Estado do Rio de Janeiro, Rio de Janeiro, 2013. 10p. Disponível em: <http://www.inea.rj.gov.br/inea/areas_contaminadas.a sp>. Acesso em: mar. 2014.

MAGALHÃES, J. S. B. Avaliação da Gestão de Sítios Contaminados por Resíduos Perigosos nos EUA, Canadá, Países Europeus e Brasil, e Exemplo de um Manual Simplificado de Avaliação de Saúde Ambiental destes Sítios para o Brasil. 2000. 95p. Dissertação (Mestrado em Ciências na área de Saúde Pública). Fundação Oswaldo Cruz, Escola Nacional de Saúde Pública, Rio de Janeiro - RJ.

MATTIASO, D. Nova Fase para Gestão de Solos Contaminados. Revista Água e Ambiente Subterrâneo (ABAS), n.14, p.18-19, Fevereiro/Março 2010.

PANAGOS, P.; LIEDEKERKE, M. V.; YIGINI, Y. \& MONTANARELLA, L. Contaminated Sites in Europe: Review of the Current Situation Based on Data Collected Through a European Network. Journal of Environmental and Public Health, 2013, article ID 158764.

RODRIGUES JR., J. J. Proposta Metodológica para Gerenciamento de Áreas Contaminadas: uma Aplicação no Estado do Rio de Janeiro. 2003. 100p. Dissertação (Mestrado em Planejamento Energético). PPE/COPPE/UFRJ, Rio de Janeiro - RJ.

SWARTJES, F. A. (Ed.) Dealing with Contaminated Sites: From Theory Towards Practical Application. Netherlands: National Institute of Public Health and the Environment (RIVM). Springer, 2011. 1114p.

SWARTJES, F. A.; RUTGERS, M.; LIJZEN, J. P. A.; JANSSEN, P. J. C. M.; OTTE, P. F.; WINTERSEN, A.; BRAND, E. \& POSTHUMA, L. State of the Art of Contaminated Site Management in The Netherlands: Policy Framework and Risk Assessment tools. Science of the Total Environment, v. 427, p. 1-10, 2012. 